\title{
Toward a practical mobile robotic aid system for people with severe physical disabilities
}

\author{
Michael A. Regalbuto, PhD; Thomas A. Krouskop, PhD; John B. Cheatham, PhD \\ Adept Technology, San Jose, CA 94086; Baylor College of Medicine, Houston, TX 77030; Department of \\ Mechanical Engineering, Rice University, Houston, TX 77251
}

\begin{abstract}
A simple, relatively inexpensive robotic system that can aid severely disabled persons by providing pick-and-place manipulative abilities to augment the functions of human or trained animal assistants is under development at Rice University and the Baylor College of Medicine. A stand-alone software application program runs on a Macintosh personal computer and provides the user with a selection of interactive windows for commanding the mobile robot via cursor action. A HERO 2000 robot has been modified such that its workspace extends from the floor to tabletop heights, and the robot is interfaced to a Macintosh SE via a wireless communications link for untethered operation. Integrated into the system are hardware and software which allow the user to control household appliances in addition to the robot. A separate Machine Control Interface device converts breath action and head or other three-dimensional motion inputs into cursor signals. Preliminary in-home and laboratory testing has demonstrated the utility of the system to perform useful navigational and manipulative tasks.
\end{abstract}

Key words: control panel, kinematics, manipulative aid, mobile robot, model-reflective command generation, personal autonomy, teleoperator, severely disabled, world modeling.

\section{INTRODUCTION}

Robotic technology is being used to provide severely physically disabled people with increased independence in home and office environments (1-6). In addition to the personal benefits of increased autonomy, possible economic

Address all correspondence and reprint requests to: Thomas $\mathrm{A}$. Krouskop, $\mathrm{PhD}$, Department of Rehabilitation, Baylor College of Medicine, 1333 Moursund Avenue, Houston, TX 77030. benefits underlie the application of robotics in the health care industry (7). Researchers at Stanford University/VA Medical Center (VAMC), Palo Alto, CA, Carnegie Mellon University, and Boeing Aerospace have developed robotic aid prototypes in the form of voice-activated workstations, in which personal items, work materials, and appliances are placed within reach of a fixed robot that can transport objects from one place to another or present objects to the user. Also, researchers at Stanford/Palo Alto VAMC developed a prototype voice-activated mobile robotic aid $(8,9)$. PRAB Command Inc. commercialized (unsuccessfully) a robotic workstation based on the system developed at Boeing.*

Widespread application of robotic technology in rehabilitation is hampered by the high cost and limited utility of the equipment, lack of reliability, frequency and technicality of maintenance, and difficulty in training people to use a particular system. The prototype robotic system under development at Rice/Baylor addresses these issues. Costs are reduced by using off-the-shelf technology wherever possible. Aside from the Machine Control Interface (MCI), a framework for a useful system was developed using a modified HERO 2000 robot, a Macintosh SE host computer, and the X-10 Powerhouse household appliance controller (cost under $\$ 8,000$ ). Utility of the system is enhanced by using equipment that is multifunctional. The MCI is designed to serve as an all-in-one control unit, capable of generating control signals for a variety of machines, including wheelchairs, a musical synthesizer, and personal

*PRAB Command, Inc.: Voice Controlled Personal Robot, news release, Kalamazoo, MI, June 1988. 


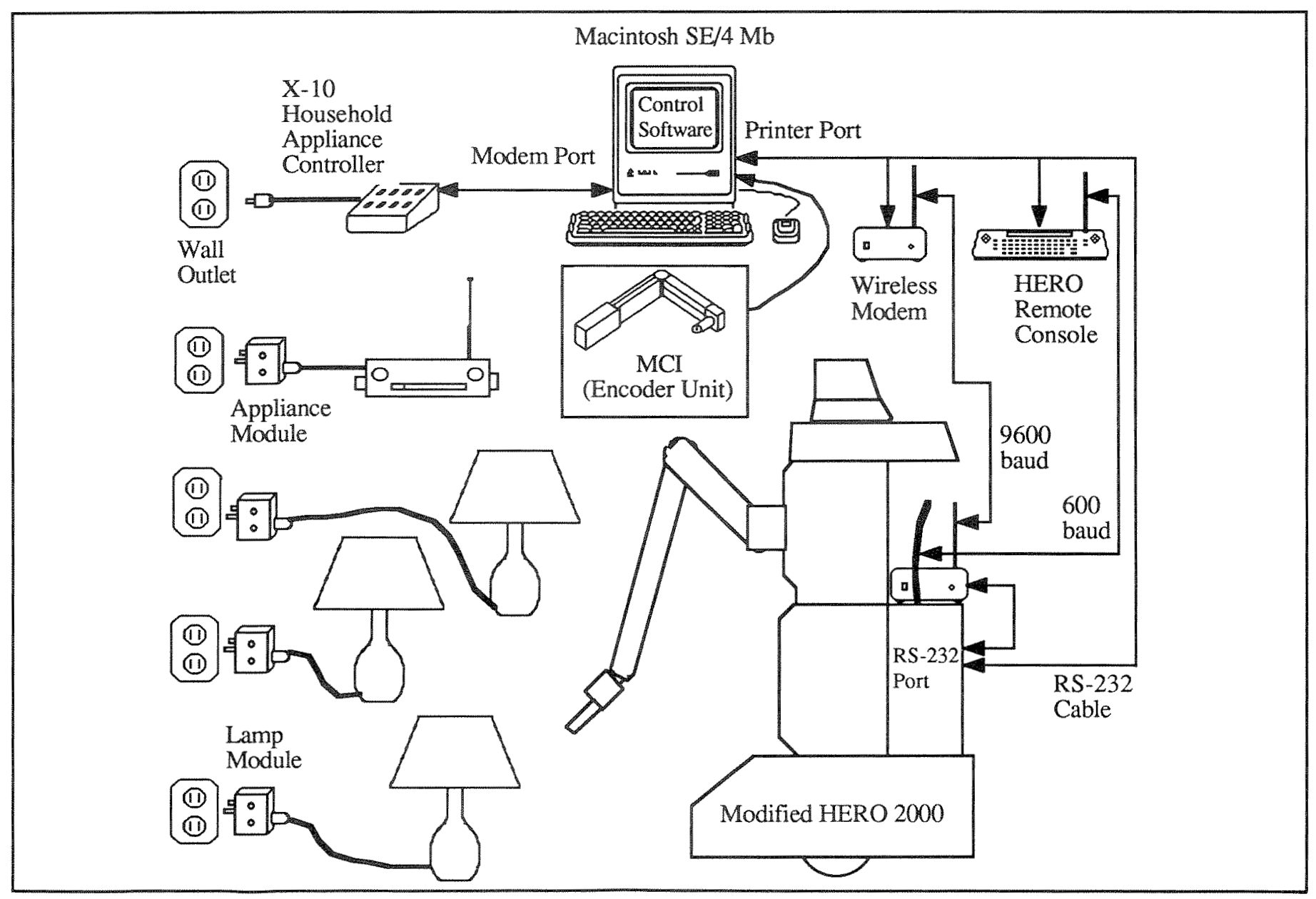

Figure 1.

Schematic of robotic aid system.

computers; thus, in the absence of the robot, the system can still serve as an environmental control unit and as a wheelchair controller. Increased hardware utility, reliability, and maintainability of the robot and its communication link have been the subject of ongoing efforts at Rice $(10,11,12)$, but will continue to be the bane of the system until better technology and support are available from manufacturers. In the meantime, many of the hardware reliability problems have been ameliorated with more robust software. Moreover, the host computer and the robot itself have intrinsic value as personal computers, and are members of two very popular brands for which a great deal of software exists. The difficulty in training users has been addressed by keeping the human/machine interface simple. Many of the elements of the Macintosh user interface, including windows, control panels, and interactive graphics, are incorporated into the robot control software, which is entirely cursor-driven. The software exists as a stand-alone application on a single high-density 3.5 inch floppy disk. The entire system boots with the signal of a single click of a mouse.

\section{METHODS AND MATERIALS}

\section{System configuration}

Figure 1 is a schematic of the robotic aid system, in which a Macintosh SE, a HERO 2000 robot, and a Powerhouse X-10 household appliance controller are integrated. The host computer forms the hub of the system, communicating with the robot and appliance controller via its programmable baud rate serial printer and modem ports. At the expense of hindering the mobility of the robot, a cable presently provides the most reliable communications link between the host and the robot. The remote console (RC) supplied with the robot allows untethered operation 


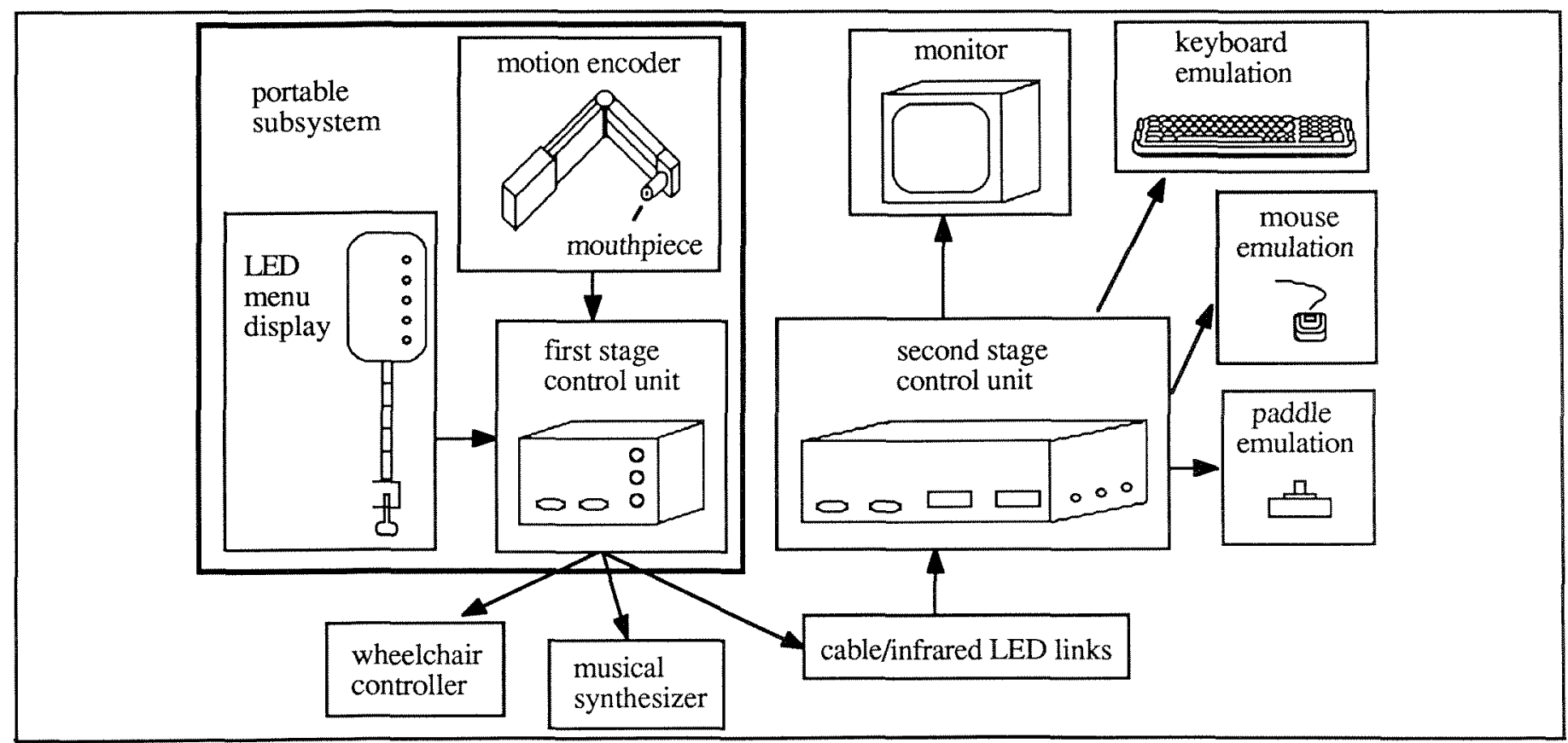

Figure 2.

Schematic of Machine Control Interface.

with a range of up to 50 feet, but at 600 baud the communications link is slow and can hamper the utility of the robot. The lack of dependable hardware handshaking in the $\mathrm{RC}$ requires extensive software error checking by communications routines on the host computer. The range and communications limitations of the console can be overcome at additional cost (several thousand dollars) by using a pair of 9600 baud wireless modems. These modems have been used with this project and several other projects where they proved to be reliable and able to solve the problems inherent with the HERO RC.

\section{Robot}

Designed for domestic and educational use, the HERO has a number of features which make it suitable for use as a domestic aid. With its 5-degree-of-freedom arm and parallel jaw gripper, the robot can manipulate payloads of up to 1 pound. Dual independent drive wheels in the base give the robot the ability to move forward and backward as well as to turn in place. An onboard 8088 microprocessor and independent motor controller processors simplify the task of programming the robot in BASIC. Arm joints are commanded directly in degrees and base movement in inches. MS-DOS can be run using an optional disk drive, hence languages other than BASIC can be supported.

Several mechanical components of the off-the-shelf robot have been modified to increase its utility. The origi- nal robot was able to reach from ground level to barely above 30 inches from the ground. By lengthening the second segment of the arm from 9 to 18 inches and raising the arm 9 inches in an extended torso section, the robot is able to reach above a tabletop height of 30 inches, with a 24-inch depth of reach at that height (10). A shockabsorbing suspension has been designed to replace the fixed suspension of the original robot (11). This modification alleviates problems with jerkiness in stopping, starting, and traversing small obstacles. A 3-degree-of-freedom wrist and a gripper with improved payload, dexterity, and sensing are currently under development (12).

Autonomous function of a robot depends on the ability of the robot to sense both its external environment and its internal state. The HERO is equipped with a suite of sensors for infrared light and sound detection, sonar range finding, temperature sensing, and low-battery sensing. The sonar and light sensors have been used in navigation routines involving obstacle avoidance, wall following, homing, and triangulation of position. By using an optional experimenter card it is reasonable to add even more sensors. For example, sonar and optical switch sensors have been designed for attachment to the robot gripper, and automated grasping routines have been implemented (13). In combination with the low-battery sensor, an infrared light detector is used in a routine for making the robot move to its charger. A speech synthesis unit, useful in providing audible feed- 


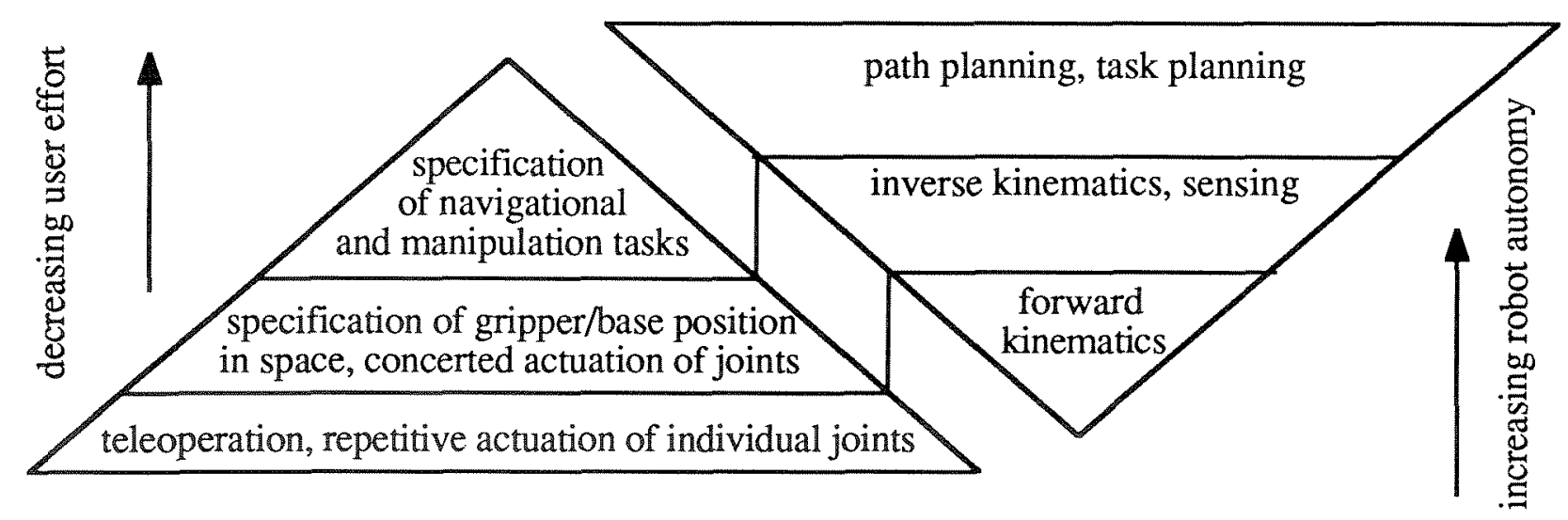

Figure 3.

Robot command hierarchy.

back to indicate that the robot has received commands, is among the various other features of the robot.

\section{Machine control interface}

The machine control interface (MCI) is a userprogrammable unit that enables interaction with a wide range of electromechanical devices using one set of userselected control inputs. The MCI is composed of a position encoder unit, a light-emitting diode (LED) menu display, and first- and second-stage control units (Figure 2). The position encoder, LED display, and first-stage control unit constitute a portable subsystem designed to be carried on the wheelchair of a user. The first-stage control unit has outputs for controlling a wheelchair and a musical synthesizer, or creating RS-232 encoded signals for controlling a computer. Using the RS-232 protocol, the first-stage control unit communicates with the second-stage over either cable or infrared links. The second-stage unit produces mouse, paddles, and keyboard outputs that can drive a separate host computer. One notable feature of the $\mathrm{MCI}$ is that its output response and sensitivity are programmed by the individual user to fit his range of motion and desired posture. As a safety feature, the MCI can detect when the encoder is "out of range" (should the encoder fall from the user's mouth), and can be programmed to respond appropriately when controlling a particular device.

In its current embodiment, the encoder unit is a threesegment, parallel-linkage arm. A pair of Hall-effect transducers is placed inside each segment, and measures the relative motion of the parts of the linkage. The encoder translates three-dimensional (3-D) head movement into three independent analog outputs and uses breath sip/puff signals to provide a fourth proportional control channel. Fitted with a mouthpiece, the encoder can be mounted on the wheelchair of the user, or directly on the user (i.e., a yoke that fits over the shoulders).
For the purpose of controlling the robot, the MCI emulates the action of a hand-held mouse. Seated comfortably in a wheelchair, the user grasps the encoder mouthpiece in his mouth, whereupon the segments of the encoder deflect in response to head motions. Side-to-side head movements are translated into horizontal movement of the cursor on the computer screen. Similarly, up-and-down nodding motions produce vertical movement of the cursor. A puff into the mouthpiece is translated into the mouse "click" action.

\section{Robot command modes}

Command modes for the robot can be classified within a hierarchy beginning with forward kinematic control, proceeding to inverse kinematic control, and ending with path and task planning (Figure 3). One layer of the hierarchy builds upon the next; high-level navigation and manipulation tasks can be decomposed into sequences of lower-level forward and inverse kinematics commands and sensing operations. The hierarchy reflects the distribution of effort between the user and the robot. As the robot becomes more autonomous by using the higher-level control, the burden on the user becomes less. The robot control software reflects this hierarchy.

\section{Control panel}

Forward kinematic commands are generated by a Teleoperator control panel (Figure 4), a computer graphic window filled with rows of "buttons" and "sliding indicators" (sliders), which when activated (clicked on) by the user, cause a joint movement command to be transmitted to the robot. In the incremental operation mode, a particular joint motion button specifies the joint and direction of movement, and the corresponding slider is set to indicate the desired amount of incremental movement of the joint. For instance, when the user clicks on the "arm dn" button with the corresponding slider set at " 15 ," the arm 


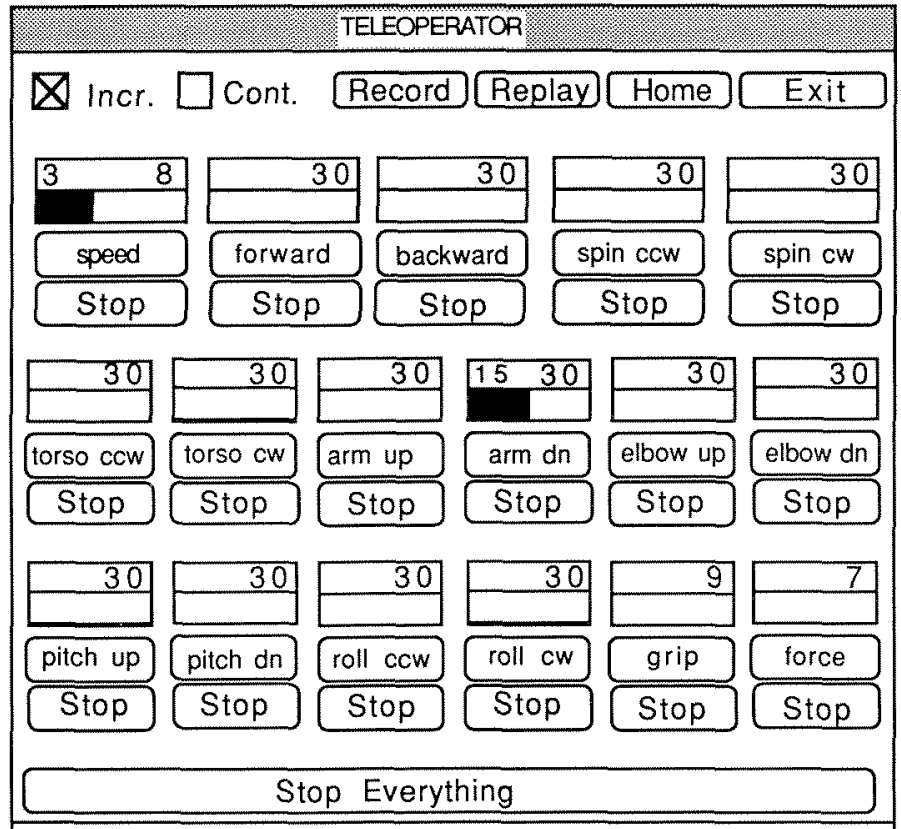

torso 0: arm 40: elbow 90: pitch 90: roll 0: grip 0: force 0: speed 3 ok

\section{Figure 4.}

Teleoperator control panel for forward kinematic control.

joint is caused to move down by 15 degrees. A "continuous" mode can be selected in which activity is initiated with a click on the joint motion button, and terminated only after a subsequent click on the corresponding "Stop" button is made, or after the joint has reached the limit of its range of motion. The individual "Stop" buttons as well as the large "Stop Everything" button provide a means for halting robot motion in an emergency situation.

\section{Inverse kinematic commands and world modeling}

Inverse kinematic commands, those that involve positioning the gripper and base of the robot with respect to a predefined global reference frame, are generated with the aid of a world model (a database containing information about the robot and its surroundings). Currently the environment of the robot is assumed to be static, structured, and indoors. Database creation and management are facilitated by means of an interactive graphics interface written in Object-Oriented LISP (Figure 5). The interface is designed to operate much like commonly available graphics applications such as MacDraft or MacDraw, possibly facilitating training of experienced Macintosh users. Using a palette of primitives which represent real-world objects, the user first creates a model of the robot and its environment. Though fully three-dimensional, the model is displayed as a two-dimensional top-down view. Selected objects appear with a graphic window and can be sized and positioned using cursor action or keyboard data entry.

Once the model environment has been given sufficient detail to define a set of task paths, commands for the real robot are generated either by selecting from a menu of possible actions, or by manipulating the model robot in a manner indicating the desired task. The robot itself is modeled as a combination of two primitive objects, a base and a gripper. Given a description of the dimensions and joint ranges of the robot, the workspace of the gripper within a horizontal plane is calculated as a function of gripper height and is displayed as a shaded region (Figure 5). The gripper object can be dragged to a new location within the workspace, resulting in the generation of commands which move the real gripper within the horizontal plane. A slide is used to specify the desired vertical position of the gripper. In an analogous manner, the base object can be dragged to a new location within the model environment, and assuming a clear path to the new location exists, a series of navigational commands will be issued to the real robot to move it to the corresponding new real location. The concept of generating commands for a real robot based on manipulation of a model robot is termed "model-reflective command generation" (14).

Because the robot itself is modeled, in theory any robot can be used in this system. In practice, the kinematics of robots are relatively easy to model, and solutions exist for numerous configurations. The major remaining tasks are those of translating and transmitting commands generated in the Teleoperator control panel and World Modeler in a syntax the particular robot understands, an endeavor that is facilitated by the modular nature of the control software.

\section{Robot localization and path planning}

In order for the robot to move about its environment autonomously, the robot must be able to establish its location and be able to chart safe courses within the environment. One method of localization uses the light sensor of the robot to detect the bearings of three or more controllable (by means of the X-10) light sources at known positions within the environment. By using the light positions as input, the robot can calculate its position and orientation by triangulation (15). Given a static environment in which the locations of all objects are known, an artificial intelligence search routine known in the literature as the $\mathrm{A}^{*}$ algorithm is used to determine a shortest safe path between the current position of the robot and a user-defined destination point $(16,17)$.

\section{Robot safety}

Safety standards in the field of industrial robotics have 


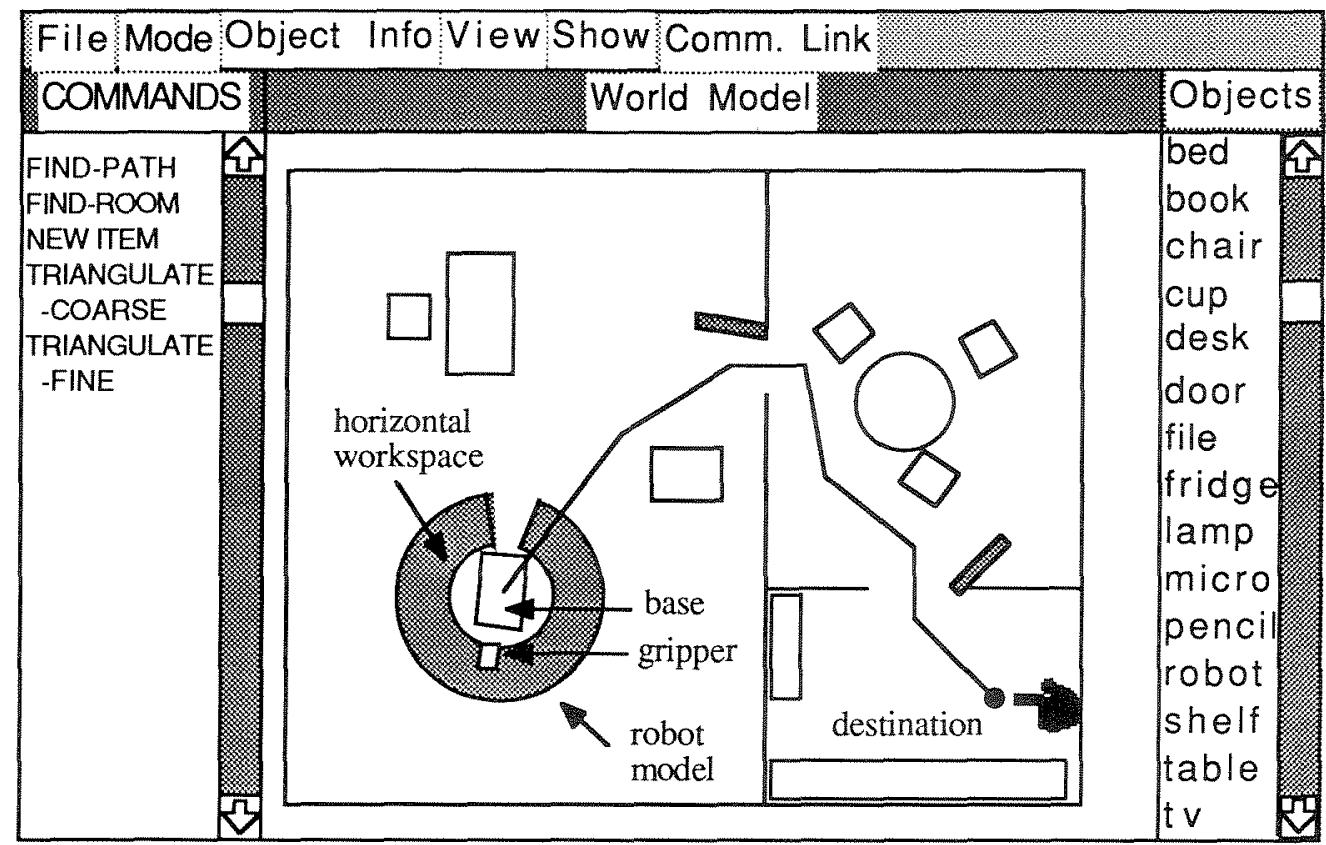

Figure 5.

World Modeler window for inverse kinematic commands and path planning.

been developed in the United States by the Robotics Industries Association (18). Industrial robots move at high speeds, can exert considerable forces, and can possibly surprise and crush an unwary operator. Therefore, safety regulations are aimed at keeping people away from a robot while it is moving and limiting any interaction by an operator with the robot under training conditions. Features such as panic buttons and dead man switches halt robot motion, while light curtains and enclosures prevent access into the workspace of the robot.

While robot safety has been the topic of recent discussions at professional society conferences such as the Rehabilitation Engineering Society of North America (RESNA), no such standards yet exist for personal robots, which by their nature are meant to interact closely with humans. In this project, a hardwired emergency stop on the $\mathrm{MCI}$ and software emergency stop buttons in the control panels provide means for halting robot motion. Also, the robot selected for this project possesses neither great speed nor great strength, so that if the robot operates away from the face of a user, the possibility for serious injury is minimal.

\section{Laboratory testing and demonstrations}

Laboratory testing of the robotic system is ongoing as new software features are written. Preliminary testing took place in the Mechanical Engineering Robotics Lab at Rice University and at The Institute for Rehabilitation and Research (TIRR) in the Texas Medical Center. The $\mathrm{MCI}$ and robot systems were demonstrated at the 1987 RESNA Conference; the MCI was used to command the robot in teleoperator mode to move cups and other small objects between the floor and a table.

Testing of the localization and path planning routines, in combination with the wireless modem link has been carried out in offices and adjoining hallways in the Mechanical Engineering building at Rice University. A world model of the entire second floor of the building was created, with details of the desks, chairs, shelves, and lamps in one of the included offices. Using the model, the position of the robot and its orientation within the office could be determined to within 6 inches and 5 degrees, although the inverse trigonometric equations used in triangulation have multiple solutions that can produce erroneous results. The robot successfully traversed paths extending from the office into the hallway. The range of the modem link was exceeded and communications lost if the robot traversed more than 100 feet from the base unit. World modeling, model reflective command generation, path planning, and untethered operation via the $\mathrm{RC}$ were demonstrated during the Space Operations Automation and Robotics (SOAR '89) conference at NASA/JSC.

\section{In-home testing}

Beginning in March 1988, the robot and MCI were placed in homes of volunteers with severe physical disabil- 
ities. At that time, the robot was tethered and none of the autonomous features had been developed. Five subjects, two women and three men, ranging in age from 20 to 46 years, used the system. All subjects had previous computer experience. Depending on scheduling and the level of interest of each subject, the robot remained in the home of a subject for a period of time ranging from 1 day to 6 weeks. The equipment was transported to the home and set up by laboratory personnel. Training was done at the time of installation, and lasted about 2 hours. The user was familiarized with the boot-up procedures for the system with the help of a two-page manual, and given a brief explanation of the Macintosh operating system, the MCI, and the control software for using the robot in the Teleoperator mode. The user was given several tasks to accomplish to assure that the system was working and the user understood the training. These tasks included opening a hinged door, moving a drink container from one surface to another, and picking up paper from the floor.

Each of the subjects was able to make the robot perform useful tasks that increased their personal autonomy, as reflected in their ability to arrange and retrieve objects in their environment without the help of assistants. As a matter of safety, tasks involving movements of the robot near the face of the user were discouraged. Such tasks include feeding and personal hygiene. The robot was designed primarily as an assist to retrieve dropped objects, to move objects in the environment (e.g., a book or a glass), and to open hinged doors. These tasks were selected from a prioritized list of tasks generated by users in the Houston area. Typically, users experimented with moving cups and other small objects about their living quarters.

The subject who used the MCI over the most extended period of time found the robot to be compatible with the MCI. Subjects found the control software easy to understand and use in conjunction with the MCI. Some users were able to operate the system using the standard mouse rather than the MCI.

During this testing, the subjects noted several areas that needed improvement. The robot was found to be cumbersome to move about the room due to its tether and to the incremental nature of commands generated in the Teleoperator mode. Those who were able to use the RC to control the robot found the RC too slow and unreliable to be useful. The time required to perform a single pickand-place task was rather long, often in the range of 5-10 minutes. Hence, the users felt that if the robot was easier to move and required fewer commands to operate, it would be a more useful tool. All users indicated that they would be interested in purchasing a robot once a simplified means of commanding the robot existed. It was on the basis of these experiments that the higher-level command software was developed. Using the World Modeler, the number of commands required for operation was reduced and tasks can now be accomplished efficiently in shorter periods of time.

The World Modeler system has been evaluated by one user, a person who had used the system the longest during the first in-home testing, and many of the problems inherent in the first generation system have been solved. The robot can be moved more quickly, typical travel times are now only 3-6 minutes for trips in an apartment. This time reduction and higher-level gripper-control function have made it possible for the user to perform pick-and-place tasks in as short a time as 2 minutes. By utilizing the robot to move items at a workstation, the user has enjoyed greater personal autonomy, and the time required to do a task is less than or equal to the time segment required to accomplish the same task using a shared attendant.

\section{DISCUSSION AND CONCLUSIONS}

The ultimate measuring stick of an assistive device is its utility to and use by the user in combination with its affordability. In developing robotic aids, various researchers have approached the question of cost/utility trade-offs from different perspectives. Some have started with high-end, industrial grade equipment and "tamed" the equipment to work in domestic or office environments. Others, such as the authors, have started with lowend, educational grade equipment and added improvements. Work must continue toward a middle ground of improved utility and decreased cost, demonstrating what can be done with all commercially available technology and, most importantly, giving end-users a range of options. In this light, several important conclusions arise from the current project:

1. Inexpensive, off-the-shelf robotic and computer technology is available that can increase the personal autonomy of the severely disabled individual. Before robotic technology will fulfill its potential for disabled people on a larger scale, a more reliable, low-cost robot must be available.

2. The Machine Control Interface is a powerful, costsaving component. The MCI provides a user-programmable all-in-one unit for controlling a variety of electromechanical devices, including wheelchairs, computers, and the robot.

3. A Macintosh or other PC can provide the framework for a system to command a mobile robot in an indoor, static, structured environment. With thoughtful planning, 
areas in the home of a severely disabled user can be made into such an environment.

4. Object-oriented LISP provides a powerful, compact structure for representing the real world and for implementing advanced problem-solving routines related to robot autonomy.

5. At the time of this writing, the HERO 2000 is no longer commercially available. Regardless, the concepts of operator control panel, world modeling, and model reflective command generation can be applied to any mobile robot.

\section{ACKNOWLEDGMENT}

This work was funded in part by a grant from the Clayton Foundation for Research and the Baylor College of Medicine.

\section{REFERENCES}

1. Cheatham JB, Regalbuto MA, Krouskop TA, Winningham DJ. A robotic system for improved living by severely disabled persons. In: Proceedings of the 1988 IEEE International Workshop on Intelligent Robots and Systems, Tokyo, October 1988. New York: Institute of Electrical and Electronic Engineers, Inc., 1988:79-82.

2. Fu C. An independent vocational workstation for a quadriplegic. In: Foulds R, ed. Interactive robotic aids-One opinion for independent living: An international perspective, monograph No. 37. New York: World Rehabilitation Fund, 1986:42-4.

3. Hammel J, Hall K, Van der Loos HMF, Leifer LJ, Perkash I. Clinical evaluation of a desktop robotic aid for severely physically disabled individuals. In: ICAART ' 88 Proceedings, Montreal, Canada, June 1988. Washington, DC: RESNA PRESS, 1988:448-9.

4. Holloway K, Leifer LJ, Van der Loos, HMF. Factors in the design and development of an interactive human-robot workstation. In: Brubaker C, ed. Proceedings of the 8th Annual RESNA Conference, Memphis, TN, June 1985. Washington, DC: RESNA PRESS 1985:268-70.

5. Lees DS, Crigler R, Van der Loos HMF, Leifer LJ. A third generation desktop robotic assistant for the severely handicapped. In: ICAART '88 Proceedings, Montreal, Canada, June 1988. Washington, DC: RESNA PRESS, 1988:450-1.
6. Leifer LJ, Michalowski SJ, Van der Loos HFM. Development of an advanced robotic aid: From feasibility to utility. In: Foulds $\mathrm{R}$, ed. Interactive robotic aids-One option for independent living: An international perspective, monograph No. 37. New York: World Rehabilitation Fund, 1986:54-7.

7. Engelhardt KG. Robots in the service of humans: Concepts and methods. In: Proceedings of the 1988 IEEE International Workshop on Intelligent Robots and Systems. Tokyo, October 1988. New York: Institute of Electrical and Electronic Engineers, Inc., 1988:815-20.

8. Van der Loos HMF, Michalowski SJ, Leifer LJ. Development of an omnidirectional mobile vocational assistant robot. In: ICAART '88 Proceedings, Montreal, Canada, June 1988:468-9.

9. Wicke R, Engelhardt KG, Awad RE, Leifer LJ, Van der Loos HMF. Evaluation of a speaker-dependent voice recognition unit as an input device for control of a robotic arm. In: Proceedings of the 6th Annual RESNA Conference, San Diego, CA, June 1983. Washington, DC: RESNA PRESS, 1983:236-8.

10. Anderson D, Lowe C, Reeme J, Struthers J, Webster W. Enhanced capabilities for a personal autonomy robot. Houston: Rice University, 1987: final report-Mech 404.

11. Badders D, Chang N, Go P, Holguin S, Lawler K, Le P. Design of RHAMBOT: Rice Handicap Aid Manipulator BOT. Houston: Rice University, 1989: final report-Mech 408.

12. Bentsen FA, McCurdy M, Finkelstein S, Porter P. Design of a dextrous hand for a mobile robot for physically disabled persons. Houston: Rice University, 1990: final report-Mech 408.

13. Fisher PB. Development of sensors and algorithms for automating robotic grasping [Thesis]. Houston: Rice University, 1989.

14. Regalbuto MA, Cheatham JB, Krouskop TA. A model-based graphics interface for controlling a semi-autonomous mobile robot. In: Proceedings of the 11th Annual International Conference of the IEEE Engineering in Medicine and Biology Society, Seattle, WA, November 1989. New York: Institute of Electrical and Electronic Engineers, Inc., 1989:910-11.

15. Regalbuto MA. A semi-autonomous mobile robot teleoperator with applications as an aid for severely handicapped people [Dissertation]. Houston: Rice University, 1990.

16. Regalbuto MA, Fisher PB, Adnan S, Norwood JD, Weiland PL. A navigation system framework for a semi-autonomous mobile robot. In: Proceedings of the Annual International Conference of the IEEE Engineering in Medicine and Biology Society, New Orleans, LA, November 1988. New York: Institute of Electrical and Electronic Engineers, Inc, 1988:1511-12.

17. Winston PH. Artificial intelligence. Reading, MA: AddisonWesley Publishing Co., Inc., 1984.

18. ANSI/RIA publication R15.06-1986. New York: American National Standards Institute, 1986. 